\title{
Cooperative Force Generation of KIF1A Brownian Motors
}

\author{
David Oriola and Jaume Casademunt \\ Departament d'Estructura i Constituents de la Matèria, Facultat de Física, Universitat de Barcelona, \\ Avinguda Diagonal 647, E-08028 Barcelona, Spain \\ (Received 21 January 2013; published 26 July 2013)
}

\begin{abstract}
KIF1A is a kinesin motor protein that can work processively in a monomeric (single-headed) form by using a noise-driven ratchet mechanism. Here, we show that the combination of a passive diffusive state and finite-time kinetics of adenosine triphosphate hydrolysis provides a powerful mechanism of cooperative force generation, implying for instance that $\sim 10$ monomeric KIF1As can team up to become $\sim 100$ times stronger than a single one. Consequently, we propose that KIF1A could outperform conventional (double-headed) kinesin collectively and thus explain its specificity in axonal trafficking. We elucidate the cooperativity mechanism with a lattice model that includes multiparticle transitions.
\end{abstract}

DOI: 10.1103/PhysRevLett.111.048103

Cooperative action of molecular motors is present in many cellular processes involving force generation and intracellular transport, which require groups of motors to team up in a dynamically self-organized manner [1-7]. A well-studied example is the clustering of motors pulling on soft cargoes such as vesicles or membrane tubes [5-11]. Under these circumstances, motors can cooperate to increase the pulling force, even though they are not rigidly attached to the cargo, thus implying a certain degree of force transmission via motor-motor interactions $[10,11]$. Recently, there has been an increasing interest in a member of the Kinesin-3 family, the kinesin KIF1A [1,12-16]. This motor works processively in vitro in a monomeric (singleheaded) form by means of a noise-driven ratchet mechanism, being a natural prototype of a Brownian motor $[12,13]$. On the other hand, KIF1A is specific of axonal transport of large synaptic vesicle precursors in neurons [1]. Although KIF1A works in a dimeric (double-headed) form in vivo, it combines a strongly bound state to the microtubule with a weakly bound diffusive state similar to that of its monomeric form [16], which is absent in conventional dimeric kinesin (i.e., the Kinesin-1 family, also referred to as KIF5), and it generically makes the individual motor inefficient and weak [12]. Although the fast motion of KIF1A seems to be explained by dimerization $[16,17]$, still the alternation with a diffusive state in vivo is at odds with the demanding conditions of axonal transport. The capacity to exert large forces along the axon would seem relevant to avoid traffic jams, a failure that has been associated with neurodegenerative diseases such as Alzheimer's [18]. Recently, it has been suggested that the existence of a diffusive state is suitable for the cooperative action of Brownian motors when they move freely and forces are unequally loaded, as in vesicle transport $[10,11]$. In such conditions, it was proposed that monomeric KIF1A could collectively achieve forces proportional to the number of motors $N$ at finite velocity. This results from a mean-field (MF) assumption that was
PACS numbers: 87.16.Wd, 05.40.-a, 87.16.Nn, 87.17.Aa

justified in the noisy environment of these motors, provided that the kinetics of adenosine triphosphate (ATP) binding was neglected [11]. On the contrary, such MF scaling strongly fails for KIF5 [4], for which the force per motor at finite velocity seems to decrease very fast for $N \sim 5$ [8]. Despite this remarkable advantage, even within MF scaling conditions, the enhanced cooperativity afforded by the diffusive state could hardly compensate the inherent weakness of the ratchet mechanism. Here, we show that the finite-time kinetics of ATP hydrolysis plays a pivotal role which enables a new powerful mechanism of collective force generation. We focus on the simplest yet most unfavorable case of monomeric KIF1A motors (hereinafter referred to as just KIF1A, unless otherwise indicated) operating in an in vitro environment and predict realistic ranges of collective forces. We conclude that the effect is in any case dramatic and that 4-6 KIF1As could well match the force of 1 KIF5, while 15 KIF1As could possibly beat any number of KIF5s. We extend the description of Refs. $[10,11]$ based on a two-state model $[13,19]$ to describe $N$ KIF1A motors moving along a onedimensional track [20]. While motors can move freely with excluded volume interactions, the external force $F$ is applied only to the foremost motor (to account for the "soft," fluidlike character of the cargo), thus leading to the formation of self-organized clusters [10,11]. Each motor labeled $\mu=1, \ldots, N$ can be in two possible states $k_{\mu}=1$, 2 corresponding to a potential $U\left(x_{\mu}, k_{\mu}\right)$. In state 1 , motors are strongly bound to the filament and subject to a sawtooth potential of periodicity $l$, asymmetry $a$, and height $U$. In the weakly bound state 2 , they undergo one-dimensional diffusion (Fig. 1). Motors switch stochastically between the two states with exponentially distributed dwell times. The decay from state 2 to 1 occurs uniformly at an average rate $\omega$, and the excitation from 1 to 2 occurs with an average rate $\omega^{\star}$ but only within a neighborhood of size $\delta \ll l$ in the potential minimum. The dwell time $1 / \omega^{\star}$ corresponds to the mean time required to capture an ATP 


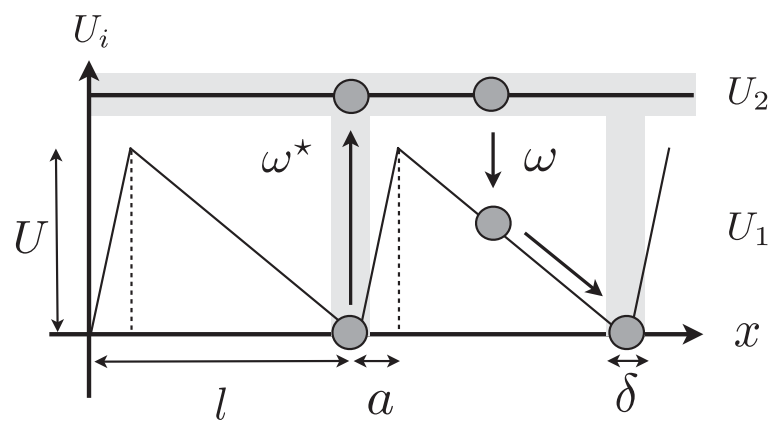

FIG. 1. Two-state ratchet description for KIF1A. Motors switch stochastically between states $k=1,2$ with potentials $U_{1}$ and $U_{2}$, respectively. Excitations are localized in regions of size $\delta$ centered at the minima of $U_{1}$, whereas deexcitations are delocalized. Gray zones depict where transitions are allowed. Small circles indicate the motor position and not its effective size, which is comparable to $l$ but typically smaller.

molecule. The existence of state 2 is the cause of the inefficiency and small stall force of a single motor. In particular, the latter is controlled essentially by $\omega[10,11]$ and is independent of $U$ and $\omega^{\star}$. However, for collective action, the diffusive state enables force transmission between the motors. On the other hand, the fraction of motors that are strongly bound to the filament will rectify the backward diffusive motion of motors in state 2 and withstand in part the external force.

The Langevin dynamics of the system reads $[10,11]$

$\lambda \dot{x}_{\mu}=-U^{\prime}\left(x_{\mu}, k_{\mu}\right)-\sum_{\nu \neq \mu} W^{\prime}\left(x_{\mu}-x_{\nu}\right)-F \delta_{1 \mu}+\zeta_{\mu}(t)$,

where $\lambda$ is a friction coefficient and $\zeta_{\mu}(t)$ is a Gaussian white noise with $\left\langle\zeta_{\mu}(t) \zeta_{\nu}\left(t^{\prime}\right)\right\rangle=2 k_{B} T \lambda \delta_{\mu \nu} \delta\left(t-t^{\prime}\right)$. Excluded volume interactions are described via a Lennard-Jones potential $W(\xi)$ of depth $\epsilon$ and motor size $\sigma$ truncated at $r_{0}=2^{1 / 6} \sigma$, where $\xi \equiv x_{\mu}-x_{\nu}$ [11]. It is worth remarking that the motor size $\sigma$ must differ

(a)

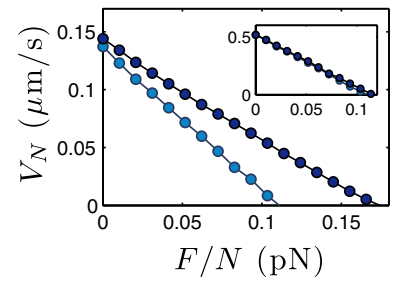

(b)

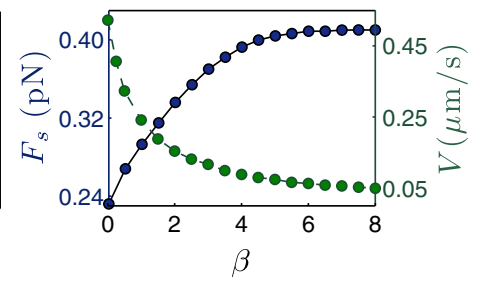

FIG. 2 (color online). (a) VF curves for $N=1$ (light blue) and $N=2$ (dark blue) for $\beta=2.5$. Inset: Same curves for $\beta=0$ showing the MF behavior. (b) Stall force and velocity at zero load for $N=2$ as a function of $\beta$. $U=20 k_{B} T, D=$ $20 \mathrm{~nm}^{2} / \mathrm{ms}, \omega=250 \mathrm{~s}^{-1}, \sigma / l=0.512, \delta / l=0.02, a / l=$ 0.2 , and $\epsilon=k_{B} T$. significantly from $l$ to enhance cooperativity [10-12]. We define the collective steady-state velocity $V_{N}(F)=\left\langle\dot{x}_{1}\right\rangle$ under a load $F$ and the collective stall force $F_{s}(N)$ of an $N$-motor cluster. The MF assumption implies $V_{N}(F)=$ $V_{1}(F / N)$ and $F_{s}(N)=N F_{s}(1)[10,11]$. Here, we will always use realistic parameter values for KIF1A extracted from the literature $[12,14]$. An important parameter will be the ratio $\beta \equiv \omega / \omega^{\star}$. Although the noise intensity is known to be large enough to validate the MF assumption if $\beta=0$ [11], we will focus on the deviations from MF for $\beta \neq 0$.

In Fig. 2, we show the velocity-force (VF) relationship for $N=1,2$. The KIF1A velocity at zero load is $0.15 \mu \mathrm{m} / \mathrm{s}$ for $\beta=2.5$, and the stall force of a single motor is $\simeq 0.1 \mathrm{pN}$, consistently with experimental values [12]. Upon the addition of a second motor, we find a remarkable enhancement of the stall force, by a factor $\simeq 3$, instead of the factor 2 implied by MF. The enhancement becomes more evident in Fig. 2(b), where the stall force of the motor pair grows with $\beta$ until saturation as a tradeoff with the velocity at zero load, which decreases similarly. For larger $N$, VF curves usually fall to very small values much before strictly vanishing, both in the Langevin and asymmetric simple exclusion process approaches [8], implying that the large $N$ scaling of $F_{s}$ differs from that of the forces at small but finite velocity. For practical purposes, it is thus convenient to define an "apparent" stall force by the condition $V_{N}\left(F_{s}\right)=V_{c}$, where we may take $V_{c} \simeq 10^{-4} \mu \mathrm{m} / \mathrm{s}$. In Fig. 3, we present the main results on the apparent stall-force dependence with $N$. We have chosen extreme values of the parameters $U$ and $\beta$ within realistic ranges compatible with experimental evidence, to estimate the lower and upper bounds of the effects predicted. We notice a steep enhancement of the (a)

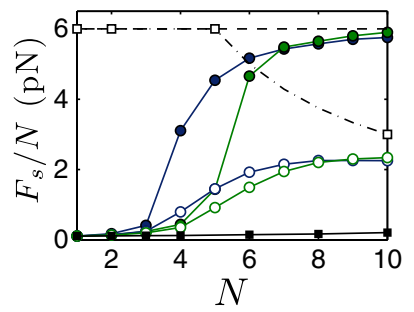

(b)

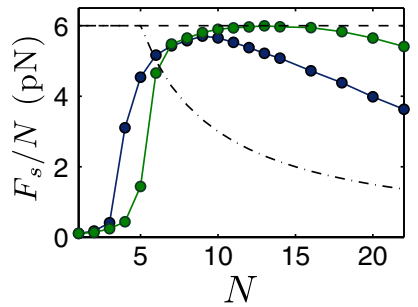

FIG. 3 (color online). Stall force per motor vs $N$. (a) Solid and open circles correspond to $U=20,10 k_{B} T$ and blue and green curves to $\beta=1,2.5$, respectively. The case $U=10 k_{B} T$ is made more unfavorable by softening the hard-core repulsion with an exponential tail of range $0.2 l$ (as in Ref. [11]). The apparent stallforce scaling for KIF5 (dash-dotted line) is extrapolated from the data of Fig. 3(c) in Ref. [8] (open squares) following 30/ $N$ for $N \geq 5$, and compared to the MF scaling for KIF5 (dashed line). The stall force of 1 KIF5 is taken as $6 \mathrm{pN}$. Solid squares indicate the MF scaling of KIF1A for $\beta=0$ and $U=20 k_{B} T$. (b) Same plot for larger $N$. The rest of the parameters are those of Fig. 2. 
force per motor at 4-5 motors, which is independent of the parameter choice. KIF1A stall forces are compared to the predictions in Ref. [8] for KIF5 in the case of repulsive interactions (open squares and dash-dotted lines). Our results show that $10 \mathrm{KIF} 1 \mathrm{~A}$ motors can achieve a stall force on the range of $20-60 \mathrm{pN}$, while the maximum force that any number of KIF5s could achieve according to Ref. [8] would be around $30 \mathrm{pN}$. As shown in Fig. 3(b), for even larger $N$, the apparent stall force per motor reaches a maximum value around $N \sim 10$ or larger, and then it is slowly decreasing because of the saturation of the apparent stall force. We notice that the apparent and strict stall forces differ significantly only for $N>5$ for KIF5 [8] and for $N \gtrsim 10$ for KIF1A (Fig. 3). The full VF curves will be discussed elsewhere [21], but it is worth remarking that the collective force enhancement is also found for intermediate velocities.

To gain further insights, it is useful to introduce an appropriate generalization of the asymmetric simple exclusion process approach $[8,22,23]$. From the Langevin picture, it is clear that the key events that enhance cooperativity beyond MF involve simultaneous motion of different motors, namely, when a motor sliding down the ratchet slope in state 1 pushes a motor in state 2 (assuming $a<\sigma<l-a)$ [10,11]. Furthermore, simulations of many motors under large forces give rise to very packed motor clusters that advance with a high degree of coordinated motion. These observations suggest that an appropriate discrete model should incorporate transitions of blocks of adjacent motors, with rules that should be grounded on the Langevin formulation. Here, we introduce such a model for the simplest and most illuminating case $N=2$ [24].

We consider a 1D lattice with each site associated to a period of the ratchet potential of length $l$ and asymmetry $a$, with two possible states. Single-motor transitions are shown in Figs. 4(a) and 4(b): in state 2, a motor labeled $\mu$ can undergo biased diffusion along the lattice with rates $u_{\mu}$ and $v_{\mu}$. Since the external load is applied only to the leading motor, we will have $u_{\mu}=(d / 2)\left(1-\delta_{1 \mu} f\right)$ and $v_{\mu}=(d / 2)\left(1+\delta_{1 \mu} f\right)$, where $f \equiv F l / k_{B} T$ is the dimensionless force and $d \equiv k_{B} T / \lambda l^{2}$ the diffusion rate. On the other hand, the motor can decay from state 2 to state 1 either at the same site or into the next one on the right [Fig. 4(b), upper panel], reflecting the distinct probability of falling into the red or green regions of the ratchet potential [Fig. 4(a)], with respective rates $q_{\mu}$ and $p_{\mu}$. Consistently with the Langevin picture, we take $p_{\mu} \equiv p=$ $(\omega / 2)(1-2 \bar{a})$ and $q_{\mu} \equiv q=(\omega / 2)(1+2 \bar{a})$, where $\bar{a} \equiv a / l$. Finally, we assume for simplicity that the excitation rate $r_{\mu}$ is essentially independent of the external load, with a dwell time in state 1 that is dominated by the time the motor needs to capture an ATP molecule, thus neglecting the sliding time along the slope $\left(r_{\mu} \simeq \omega^{\star}\right)$. These assumptions are realistic for KIF1A for small loads (a)

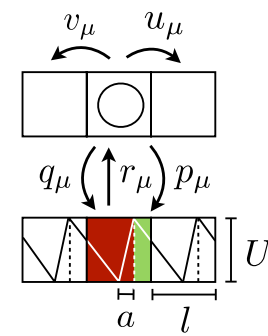

(c)

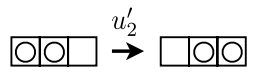

(e)

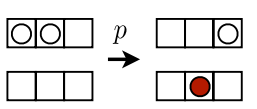

(b)
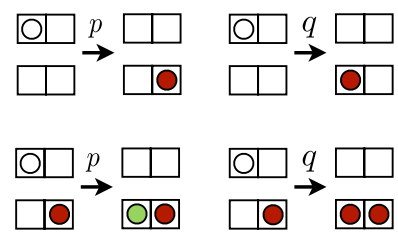

(d)

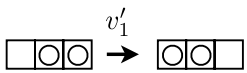

(f)

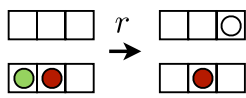

FIG. 4 (color online). Lattice model. (a) Elementary transitions in the lattice model and their correspondence with the ratchet picture. (b) $p$ and $q$ decays reflecting the probability of falling into the green or red regions shown in (a). If the second motor attempts a $p$ decay from state 2 while the next site is occupied in state 1, the first is trapped in the "green" state (state 3 in the text). (c),(d) Coordinated diffusion of two motors in state 2. (e) "Down-push": The second motor makes a $p$ decay and pushes the first one. (f) "Up-push": The first motor excites and it is pushed by the second one in state 3 .

$f \ll f_{r}$, where $f_{r} \equiv U /(1-\bar{a}) k_{B} T$ and $d \gg p$ [12]. For isolated motors, this model is a simple extension of those of Refs. [14,25], including unequal loading. In our model, however, we relax the simple exclusion principle and allow for coordinated steps when one motor attempts to move into an occupied site. Two adjacent motors in the diffusive state 2 will thus be allowed to move together one step forward or backward with hopping rates $u_{2}^{\prime}=u_{1} / 2$ and $v_{1}^{\prime}=v_{1} / 2$, respectively [Figs. 4(c) and 4(d)]. The crucial processes are those in which adjacent motors undergo a change of state, in particular, the $p$ transitions, which are the ones that contribute to motion and power generation. Consider the case when the second motor attempts a $p$ decay from state 2 while the next site is occupied by the first motor. If the latter is also in state 2 , then we must allow the simultaneous step forward of both. This is what we call a "down-push" [see Fig. 4(e)] and it corresponds to the cooperativity mechanism identified in Refs. [10,11]. Only if $\beta \neq 0$, a new situation occurs when the $p$ decay is attempted while the first motor is occupying the adjacent site in state 1 [Fig. 4(b), lower panel]. As suggested by the Langevin dynamics, the proper way to model this case is that the second motor must wait until the first one is excited, and then both will move forward together on step. This is the new key feature that is introduced by the finite dwell time. In order to keep a Markovian description, without memory effects, this situation may be handled in practice by defining a new state 3 , as the waiting state of the second motor after a $p$ decay when the adjacent state is occupied in state 1 . This state is depicted in green in Figs. 4(b) and 4(f) and has no counterpart in the 
single-motor problem or when hydrolysis dwell time is neglected. Such an event may be called an "up-push" [Fig. 4(f)] and provides the new mechanism required to enhance cooperativity beyond MF. To pursue this model analytically, we first consider the case $N=1$. If we define the probability of finding the motor in states $s=1,2$ as $\sigma_{s}(t)$, then we have

$$
\dot{\sigma}_{1}=\left(q_{1}+p_{1}\right) \sigma_{2}-r_{1} \sigma_{1} ; \quad \dot{\sigma}_{2}=-\dot{\sigma}_{1} .
$$

In the steady state, we find $\sigma_{1}^{\text {ss }}=\beta /(1+\beta)$ and $\sigma_{2}^{\text {ss }}=$ $1 /(1+\beta)$. Since the motor can only advance from state 2 , we have $V_{1}(f)=l \sigma_{2}^{\text {ss }}\left(u_{1}+p-v_{1}\right)$, that is,

$$
V_{1}(f)=\frac{v_{d}}{1+\beta}\left(f_{1}-f\right),
$$

where $v_{d} \equiv l d$ and $f_{1} \equiv p / d$ is the dimensionless stall force $f_{s}(1)=f_{1}$. The linear VF curve coincides with the prediction of the Langevin model for large noise strength [11]. The case $N=2$ can be easily solved numerically, but some analytical approximations are illuminating [26]. We define a generic configuration state $\mathcal{C}=\left\{s_{1}, s_{2}, n\right\}$ by the conditions of the first and second motors being, respectively, in states $s_{1}$ and $s_{2}$, having $n$ vacant sites in between. The space of possible configurations can be enumerated as $\{1,1, n\},\{1,2, n\},\{2,1, n\}$, and $\{2,2, n\}$ with $n \geq 0$ plus the configuration $\{1,3,0\}$, the only one that involves state 3 . We then define a decoupling approximation as $P(\mathcal{C}, t) \simeq$ $\sigma_{s}(t) \tau_{s}(t) \rho_{n}(t)$ for $s=1, \quad 2, \quad n \geq 0$ while keeping $P(\{1,3,0\}, t) \simeq \tau_{3}(t)$, where $\tau_{s}(t)$ is the probability of finding the second motor at state $s$ and $\rho_{n}(t)$ is the vacant probability distribution within our approximation. This decoupling neglects correlations between spatial and internal degrees of freedom but is not quite a MF ansatz due to specific treatment of the configuration $\{1,3,0\}$. As in the MF ansatz, though, it is expected to be reasonable for large noise strength, i.e., large diffusivity and relatively small forces. The dynamics of $\tau_{s}(t)$ and $\rho_{n}(t)$ can be solved and it yields the steady-state solutions $\rho_{0}^{\text {ss }}=f /\left(1+f_{1}+f / 2\right)$, $\tau_{2}^{\mathrm{ss}}=1 /\left[1+\beta\left(1+c \sigma_{1}^{\mathrm{ss}} \rho_{0}^{\mathrm{ss}}\right)\right], \quad \tau_{1}^{\mathrm{ss}}=\beta \tau_{2}^{\mathrm{ss}}, \quad$ and $\quad \tau_{3}^{\mathrm{ss}}=$ $\beta c \sigma_{1}^{\text {ss }} \rho_{0}^{\text {ss }} \tau_{2}^{\text {ss }}$, where $c=(1 / 2)(1-2 \bar{a})$ [26]. The VF curve can be written explicitly in terms of the transitions involving the motion of the first motor and their respective probabilities [26]. Inserting the above approximate values, we get

$$
\begin{aligned}
V_{2}(f) & \simeq \frac{v_{d}}{1+\beta}\left[f_{1}-f+\rho_{0}^{\mathrm{ss}} g(f)\right], \\
g(f) & =\tau_{2}^{\mathrm{ss}}\left(f_{1}-\frac{f}{2}\right)+f_{1} \tau_{1}^{\mathrm{ss}}+\frac{1}{2}(1+f) .
\end{aligned}
$$

The exact numerical simulation of the problem shows that this approximation actually yields an underestimation of $V_{2}(f)$ [26]. At zero load $\rho_{0}^{\text {ss }}(f=0)=0$ and from Eq. (4), we recover the single-motor velocity. The first term in $g(f)$ accounts for the down-push transitions [Fig. 4(e)] considered in Refs. [10,11]. Note that this contribution is decreasing with $\beta$, and it is negative for $f \geq 2 f_{1}$, so it cannot cause the stall-force enhancement beyond the MF value. Conversely, the second term, which vanishes if $\beta=0$ (i.e., $\tau_{3}^{\text {ss }}=0$ ), comes from the up-push transition [Fig. 4(f)], and it grows with $\beta$ up to a saturation value. This term, which originates from the finite-time kinetics of ATP hydrolysis, is the only one that allows for an increase of the stall force beyond MF. Interestingly, a shortage of ATP results in a decrease of velocity but, up to a point, in a significant increase of the strength of the motor pair. The last term is due to purely excluded volume interactions between the motors, that is, coming solely from the interaction potential $W$, as opposed to the two first terms which are due to the filament force (i.e., the potential $U_{1}$ ). For $\beta=0$, this last term yields the MF stall force of two motors $f_{s}(2)=2 f_{1}$. In the limit $\beta \gg 1$, the approximate stall force is indeed larger than the MF prediction and takes the form [26]

$$
\frac{f_{s}(2)}{2 f_{s}(1)} \geq \frac{1+f_{1}}{1-f_{1}}>1 \text {. }
$$

We have shown that the diffusive nature of KIF1A is particularly adapted to cooperative force generation. In particular, the introduction of finite-time hydrolysis in the chemical cycle may contribute significantly to enhance the collective force. The combination of several motors enables a switch from a noise-driven mechanism with a low force scale $\left(\sim k_{B} T l / l_{D}^{2}\right.$, with the diffusion length $l_{D}=$ $\sqrt{2 D / \omega})$ to a filament-binding mechanism with a high force scale $(\sim U / l)$. Our results predict that monomeric KIF1A could outperform KIF5 when tens of motors team up spontaneously. This prediction can be directly tested in tube-pulling experiments in vitro [27].

We acknowledge financial support under Projects No. FIS2010-21924-C02-02 and No. 2009-SGR-014. D. O. also acknowledges a FPU grant from the Spanish Government.

[1] N. Hirokawa, Y. Noda, Y. Tanaka, and S. Niwa, Nat. Rev. Mol. Cell Biol. 10, 682 (2009).

[2] E. L.F. Holzbaur and Y.E. Goldman, Curr. Opin. Cell Biol. 22, 4 (2010).

[3] J. Howard, Mechanics of Motor Proteins and the Cytoskeleton (Sinauer, Sunderland, MA, 2001).

[4] A. K. Rai, A. Rai, A. J. Ramaiya, R. Jha, and R. Mallik, Cell 152, 172 (2013).

[5] G. Koster, M. VanDuijn, B. Hofs, and M. Dogterom, Proc. Natl. Acad. Sci. U.S.A. 100, 15583 (2003).

[6] C. Leduc, O. Campàs, K. B. Zeldovich, A. Roux, P. Jolimaitre, L. Bourel-Bonnet, B. Goud, J.-F. Joanny, P. Bassereau, and J. Prost, Proc. Natl. Acad. Sci. U.S.A. 101, 17096 (2004).

[7] C. Leduc, O. Campàs, J.-F. Joanny, J. Prost, and P. Bassereau, Biochim. Biophys. Acta 1798, 1418 (2010). 
[8] O. Campàs, Y. Kafri, K. B. Zeldovich, J. Casademunt, and J. F. Joanny, Phys. Rev. Lett. 97, 038101 (2006).

[9] O. Campàs, C. Leduc, P. Bassereau, J. Casademunt, J.-F. Joanny, and J. Prost, Biophys. J. 94, 5009 (2008).

[10] J. Brugués and J. Casademunt, Phys. Rev. Lett. 102, 118104 (2009).

[11] J. G. Orlandi, C. Blanch-Mercader, J. Brugues, and J. Casademunt, Phys. Rev. E 82, 061903 (2010).

[12] Y. Okada, H. Higuchi, and N. Hirokawa, Nature (London) 424, 574 (2003).

[13] Y. Okada and N. Hirokawa, Science 283, 1152 (1999).

[14] K. Nishinari, Y. Okada, A. Schadschneider, and D. Chowdhury, Phys. Rev. Lett. 95, 118101 (2005).

[15] M. Kondo, Y. Takei, and N. Hirokawa, Neuron 73, 743 (2012).

[16] J. W. Hammond, D. Cai, T. L. Blasius, Z. Li, Y. Jiang, G. T. Jih, E. Meyhofer, and K.J. Verhey, PLoS Biol. 7, e1000072 (2009).

[17] M. Tomishige, D. R. Klopfenstein, and R. D. Vale, Science 297, 2263 (2002).

[18] G. B. Stokin et al., Science 307, 1282 (2005).
[19] F. Jülicher, A. Ajdari, and J. Prost, Rev. Mod. Phys. 69, 1269 (1997).

[20] For simplicity, we neglect attachment or detachment kinetics between the track and the cargo.

[21] D. Oriola and J. Casademunt (unpublished).

[22] A. Parmeggiani, T. Franosch, and E. Frey, Phys. Rev. Lett. 90, 086601 (2003).

[23] M. R. Evans, Europhys. Lett. 36, 13 (1996).

[24] An extension of the model to an arbitrary number of motors can be done with qualitatively similar results to the Langevin model.

[25] D. Chowdhury, Physica (Amsterdam) 372A, 84 (2006).

[26] See Supplemental Material at http://link.aps.org/ supplemental/10.1103/PhysRevLett.111.048103 for detailed derivations and Monte Carlo simulations of some results described in the text.

[27] During revision, preliminary experiments have confirmed that KIF1A motors can indeed extract tubes, thus validating our prediction; D. Oriola, S. Roth, M. Dogterom, and J. Casademunt (unpublished). 